\title{
Inhaltsverzeichnis Band 1
}

Vorwort - V

Inhaltsübersicht — VII

Einleitung: Konzeption und Thesen - 1

\section{Teil 1 Grundlagen - 37}

1 Forschungsstand: Narrative der NS-Kirchengeschichtsschreibung - 39

2 Theorie und Methodik — 61

2.1 Theorie: Grundannahmen -63

2.1.1 Sozialtheorie: Individuen vs. Strukturen und Überzeugung vs. Handlungen -63

2.1.2 Gesellschaftstheorie: Individuen vs. Institutionen und Kollektive -74

2.1.3 Erkenntnistheorie: Kollektivgeschichte und Handlungstheorie - 81

2.1.4 Geschichtsverständnis und Vermittlungsansatz - $\mathbf{8 8}$

2.2 Methodik: Studiendesign -91

2.2.1 Kollektivbiografie $-\mathbf{9 2}$

2.2.2 Typologisierungen -93

2.2.2.1 Erstellung der personalen NS-Typologie und Klassifikationspraxis - 93

2.2.2.2 Erstellung der NS-Handlungstypologie - 106

2.2.3 Untersuchung der NS-Überzeugung — 111

2.2.3.1 Generierung und Validierung von NS-ÜberzeugungsIndikatoren - 116

2.2.3.2 Modell zur Messung der NS-Überzeugung — 129

2.2.4 Auswertungen - 149

2.2.4.1 Auswertung der personalen Typologie - 149

2.2.4.2 Auswertung der Handlungstypologie - 153

2.2.5 Methodologische Reflexion $-\mathbf{1 6 0}$

3 Quellen - 171

4 Hinweise zur Lesart der numerischen Daten — 193

5 Datenbank - 211 


\section{Teil 2 Personale NS-Typologie -213}

1 Qualitative Auswertung: Zehn prototypische NS-Biogramme - 219

I NS-Konsens: Zuneigung/Kollaboration - 223

(1) NS-Aktivismus: Zuneigung und radikales Engagement BOYE GEHRCKENS - 225

(2) NS-Solidarität: Zuneigung/aktive Zusammenarbeit AdOLPH PLATH -236

II NS-Konsens bei innerkirchlicher Autonomiebestrebung - 241

(3) NS-Aktivismus und Selbstbehauptung - WALTER RUSTMEIER - 243

(4) NS-Solidarität und Selbstbehauptung - KURT LUCHT - 251

III Konsensfreie innerkirchliche Autonomiebestrebung - 255

(5) Selbstbehauptung - HARALD TORP - 256

IV Zwischen Konsens und Dissens: Politisch-ideologische Ambivalenzen - $\mathbf{2 5 8}$

(6) Synchrone und diachrone Mischformen - PETER HÖHNKE - 262

V NS-Dissens: Abneigung/Opposition - $\mathbf{2 6 8}$

(7) Resistenz: Abneigung/moderate Opposition HeINRICh PETERSEN - 272

(8) Widerstand: Abneigung und Fundamentalopposition FRIEDRICH SLOTTY — 279

VI Weitere Positionierungsformen -295

(9) Opfer - ERnSt GLOYER - 298

(10) Nicht zuordenbar - Johann BLOdAu - 309

2 Quantitative Auswertung: Kollektive Häufigkeitsverteilungen - 313

2.1 NS-Positionierungsformen -314

2.2 Klassifikations-Chiffren - 336

2.3 NS-Überzeugung und Überzeugungshandeln — 346

2.4 Kirchenpolitische Zugehörigkeit und Generationalität — 363 


\section{Inhaltsverzeichnis Band 2}

Teil 3 NS-Handlungstypologie - 393

A) NS-Konformität -403

A1) Praktizierte NS-Konformität - 404

I NS-Kooperation - 406

I.1 Politische NS-Kooperation - $\mathbf{4 0 7}$

(1) NSDAP-Mitgliedschaft -416

(2) SA-Mitgliedschaft $-\mathbf{4 2 5}$

(3) SS-Mitgliedschaft -433

(4) Mitgliedschaft in anderen NS-Organisationen und Verbänden - 435

(5) Mitgliedschaft in antisemitischen und antidemokratischen Nicht-NS-Organisationen - 438

I.2 Kirchenpolitische NS-Kooperation - $\mathbf{4 4 0}$

(6) DC-Mitgliedschaft -441

(7) Mitgliedschaft in der Deutschkirche - 454

(8) Mitgliedschaft im Eisenacher ,EntjudungsInstitut' -457

II NS-Engagement $-\mathbf{4 6 0}$

(9) NS-Engagement vor $1933-464$

II.1 Politisches NS-Engagement $-\mathbf{4 7 6}$

(10) NSDAP-Ämter, Staatsämter/Aufgaben — 481

(11) NSDAP-Tätigkeit - 485

(12) SA-Ämter/-Ränge - 496

(13) SA-Tätigkeit -498

(14) SS-Ämter/-Ränge - 507

(15) SS-Tätigkeit - $\mathbf{5 0 8}$

(16) Ämter/Ränge in anderen NS-Organisationen und Verbänden - $\mathbf{5 1 2}$

(17) Tätigkeit in anderen NS-Organisationen und Verbänden - $\mathbf{5 1 5}$

(18) Ämter in antisemitischen/antidemokratischen Nicht-NS-Organisationen - $\mathbf{5 2 2}$

(19) Tätigkeit in antisemitischen/antidemokratischen Nicht-NS-Organisationen - $\mathbf{5 2 4}$

(20) Denunziationen - $\mathbf{5 3 0}$

II.2 Kirchenpolitisches NS-Engagement $-\mathbf{5 3 4}$

(21) DC-Ämter - $\mathbf{5 4 3}$

(22) DC-Tätigkeit - $\mathbf{5 4 5}$

(23) Tätigkeit für die Deutschkirche - 555 
(24) Tätigkeit für das Eisenacher ,Entjudungs-Institut ‘ $-\mathbf{5 6 0}$

(25) Eintreten für die Neuausrichtung der Kirchen nach dem NS-Staat $-\mathbf{5 6 2}$

(26) Ausgleichsbestrebungen: Eintreten für die Mitte - $\mathbf{5 7 4}$

II.3 Individuelles NS-Engagement im Rahmen des

Pfarramtes $\mathbf{5 8 2}$

II.3.1 Positive NS-Bezugnahmen - $\mathbf{5 8 7}$

(27) Mündliche Lobpreisungen Hitlers und Einschwörung auf den NS-Staat $\longrightarrow \mathbf{5 8 9}$

(28) Schriftliche Lobpreisungen Hitlers und Einschwörung auf den NS-Staat -634

(29) Proaktive NS-Zitationen - 655

(30) Defensiv-reaktive NS-Zitationen - 666

II.3.2 Propagierung von NS-Ideologie $-\mathbf{6 8 0}$

(31) Nationalprotestantismus -685

(32) Nationalismus - 705

(33) Homogene Volksgemeinschaft, Antiliberalismus und Führerprinzip - $\mathbf{7 1 9}$

(34) NS-Arbeitsideologie - 742

(35) Germanismus - 754

(36) Heroismus und Männlichkeits-Ethos - 769

(37) Bellizismus - 782

(38) Verurteilung der Weimarer Republik - $\mathbf{8 1 7}$

(39) Antikommunismus - 846

(40) Antijudaismus - 867

(41) Christlicher Antisemitismus - $\mathbf{8 9 5}$

(42) Rassismus und rassistischer Antisemitismus - 919

(43) Rassismus bei betontem Glaubensprimat - 959

(44) Lebensraumideologie - 976

(45) Eugenik und ,Euthanasie“

(46) Sozialdarwinismus - 995

(47) Weitere NS-ideologische Bezugnahmen - 1005

II.3.3 Äußere Formen der kirchlichen NS-Konformität — 1017

(48) NS-Gottesdienste - $\mathbf{1 0 2 0}$

(49) NS-Formationen im Gottesdienst und bei kirchlichen Veranstaltungen $-\mathbf{1 0 3 7}$

(50) Übernahme von NS-Symbolen - 1049

(51) NS-Personalpolitik kirchlicher Funktionsträger - 1059

III Umsetzung von kirchlichen und staatlichen NS-Vorgaben - 1069

(52) Ausstellung von, Ariernachweisen“ 1071

(53) NS-Kirchenbeflaggung - 1083

(54) ,Volk und Führer' im Kirchengebet 1090 
(55) Vereidigung auf Adolf Hitler - $\mathbf{1 1 0 0}$

(56) Reglementierte Anwendung des Hitlergrußes - 1104

A2) Innere NS-Konformität: NS-Zustimmung - 1111

I Weiche Zustimmungsindikatoren - $\mathbf{1 1 1 5}$

(57) Unvollständige theologische Ausbildung — 1117

(58) Landeskirchliche Beförderungen und Ämterübertragungen 1933-1945 - 1130

(59) Übertragene NS-Ämter und Aufgaben — 1137

(60) Freiwillige Meldung zum Militär 1933-1945 — 1142

(61) Karriere in der Wehrmacht — 1147

(62) Nach Kriegsende zur Entlassung vorgesehen - 1155

(63) Ideologisches NS-Engagement vor $1933-1159$

(64) NSDAP-Mitgliedschaft 1933-1945 - 1164

(65) Denunziationen - 1175

(66) Radikal NS-konforme Führung des Pfarramtes — 1177

(67) Hitlergruß im innerkirchlichen Schriftverkehr — 1185

(68) Bejahung von NS-Ideologie - 1192

(69) [NS-Kindernamensgebung 1930-1945] — 1213

II Starke Zustimmungsindikatoren - $\mathbf{1 2 1 7}$

(70) NSDAP-Mitgliedschaft vor dem 30.1. oder nach dem

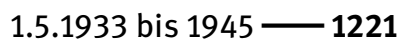

(71) Andere NS-Beitritte vor dem 30.1.1933 - 1225

(72) Parteipolitisches und/oder propagandistisches NSEngagement vor $1933-\mathbf{1 2 2 8}$

(73) Radikale politische NS-Kooperation in der NSDAP bis 1945 , SA, SS - 1232

(74) Mitgliedschaft in und/oder Engagement für die Deutschkirche - $\mathbf{1 2 4 0}$

(75) Radikales DC-Engagement - $\mathbf{1 2 4 3}$

(76) Radikales politisches NS-Engagement — 1247

(77) Anwesenheit bei Reichsparteitagen der NSDAP in Nürnberg 1253

(78) Bekundete NS-Zustimmung - 1255

(79) Bescheinigte NS-Zustimmung - 1282

(80) Entnazifizierungskategorie I bis IV - 1296

(81) NS-bezogene Sanktionen nach Kriegsende — 1302 


\section{Inhaltsverzeichnis Band 3}

\section{Teil 3 NS-Handlungstypologie (Fortsetzung) — 1319}

B) Innerkirchliche NS-Nonkonformität: Selbstbehauptung - Charakteristika des ,Kirchenkampfes' 1321

I BK-internes Wirken - Vorbedingungen des

,Kirchenkampfes' 1329

(82) BK-Mitgliedschaft 1329

(83) BK-Ämter -1330

(84) BK-Tätigkeit — 1331

II Eintreten gegen die kirchliche Selbstgleichschaltung durch die DC - ,Kirchenkampf‘ der BK im engeren Sinn - 1340

(85) Verweigerung und Kritik gegenüber DC-beherrschten kirchlichen Organen und Funktionsträgern - 1343

(86) BK-konforme Einflussnahmen auf die kirchliche Personalgestaltung - $\mathbf{1 3 6 2}$

(87) Eintreten gegen die Organisation ,Deutsche Christen' - 1370

III Innerkirchliche Verteidigung der Institution Kirche gegen Angriffe und Einflussnahmen von außen - ,Kirchenkampf‘ im weiteren Sinn 1376

(88) Eintreten gegen Deutschkirche und Neuheidentum — 1378

(89) Eintreten für die Wahrung der kirchlichen Unabhängigkeit vom NS-Staat 1409

(90) Verweigerungen kirchenbezogener NS-Vorgaben — 1430

C) Politisch-ideologische NS-Nonkonformität — 1443

C1) Praktizierte politisch-ideologische NS-Nonkonformität — 1453

(91) NS-Gegnerschaft vor $1933-\mathbf{1 4 5 8}$

I Gesellschaftspolitische Verweigerungen - 1462

(92) Private Verweigerungen gesellschaftspolitischer NS-Vorgaben - 1464

(93) Öffentliche Verweigerungen gesellschaftspolitischer NS-Vorgaben - 1466

II Protest gegen kirchenfeindliche NS-Elemente - 1472

(94) Protest gegen die Kirchenfeindlichkeit einzelner NS-Funktionäre - 1475

(95) Protest gegen kirchenfeindliche NS-Strömungen und NS-Maßnahmen - 1481

(96) Freiwilliger NS-Austritt -1494

III Nichtkirchenbezogene Kritik an NS-Führung und NS-Ideologie - 1497

(97) Kritik an NS-Führung und NS-Politik 1500 
(98) Eintreten gegen Judenfeindlichkeit und Rassismus - 1506

(99) Eintreten gegen Eugenik und ,Euthanasie“ 1522

(100) Eintreten gegen Sozialdarwinismus - 1524

(101) Eintreten gegen Militarismus und Krieg - 1525

(102) Weitere NS-nonkonforme Handlungen - 1530

C2) Innere politisch-ideologische NS-Nonkonformität:

\section{NS-Ablehnung - 1536}

I Weiche Ablehnungsindikatoren -1543

(103) Konflikt mit NS-Instanzen - 1544

(104) Parteiausschluss - 1559

(105) Versetzung und/oder Beurlaubung 1933-1945 - 1569

(106) Ablehnung von NS-Ideologemen - 1577

(107) Bekundete partielle NS-Distanz 1930-1945 - 1582

(108) Nichtverwendung des Hitlergrußes im innerkirchlichen Schriftverkehr - $\mathbf{1 5 9 2}$

II Starke Ablehnungsindikatoren - 1596

(109) NS-staatliche Strafen - 1600

(110) Entlassung aus Amt/Dienst 1933-1945 - 1603

(111) Freiwilliger NS-Austritt $-\mathbf{1 6 0 7}$

(112) Radikale praktizierte politisch-ideologische NS-Nonkonformität - 1611

(113) Bekundete NS-Ablehnung 1930-1945-1616

(114) Bescheinigte NS-Ablehnung 1930-1945 - 1620

A-C) Positionsänderungen -1631

(115) NS-konforme Positionierungsänderung - 1636

(116) NS-nonkonforme Positionierungsänderung - 1638

D) Einschränkungen und Sanktionen - 1645

D1) (Kirchen-)Politisch motivierte Einschränkungen und Sanktionen — 1655

I NS-staatliche/parteiliche Einschränkungen

und Sanktionen - 1655

(117) Moderate NS-staatliche/parteiliche Einschränkungen und Sanktionen - 1659

(118) Erhebliche NS-staatliche Sanktionen — 1667

II Landeskirchliche Einschränkungen und Sanktionen — 1672

(119) Moderate landeskirchliche Einschränkungen und Sanktionen - 1677

(120) Erhebliche landeskirchliche Sanktionen — 1686

D2) Rassenideologisch motivierte Einschränkungen und Sanktionen - 1692

(121) Rassenideologisch bedingte Einschränkungen - 1692

(122) Erhebliche rassenideologisch bedingte Sanktionen — 1693 
Teil 4 Verzeichnisse -1699

Abkürzungsverzeichnis - 1701

Abbildungsverzeichnis - 1705

Quellen- und Literaturverzeichnis - 1723

Pastorenverzeichnis - 1767 